\title{
ChemComm
}

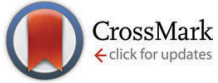

Cite this: Chem. Commun., 2016, 52, 10996

Received 28th June 2016

Accepted 11th August 2016

DOI: $10.1039 / c 6 c c 05392 k$

www.rsc.org/chemcomm

\section{An exploration of the reactivity of singlet oxygen with biomolecular constituents $\dagger$}

\author{
Barbara Marchetti ${ }^{a}$ and Tolga N. V. Karsili*ab
}

The thermal reaction between biomolecules and singlet oxygen $\left({ }^{1} \mathrm{O}_{2}\right)$ is important for rendering the genetic material within toxic cells inactive. Here we present results obtained from state-of-the-art multi-reference computational methods that reveal the mechanistic details of the reaction between ${ }^{1} \mathrm{O}_{2}$ and two exemplary biomolecular systems: guanine (Gua) and histidine (His). The results highlight the splitting of the doubly degenerate ${ }^{1} \Delta_{g}$ state of $\mathrm{O}_{2}$ upon complexation and the essentially barrierless potential energy profile of the thermally allowed cycloaddition reaction when the $\mathrm{O}_{2}$ molecule is in its lower energy ${ }^{1} \Delta_{\mathrm{g}}$ state.

The highest occupied molecular orbitals of the ground state electronic configuration of molecular $\mathrm{O}_{2}\left(\mathrm{X}^{3} \Sigma_{\mathrm{g}}{ }^{-}\right)$are a degenerate pair of $\pi^{*}$ anti-bonding orbitals, each of which contains an odd electron. This manifests in an uncommon triplet spin multiplicity (henceforth ${ }^{3} \mathrm{O}_{2}$ ) in the electronic ground state. Under ambient conditions, ${ }^{3} \mathrm{O}_{2}$ is kinetically inert towards most molecules with the 'usual' singlet ground state spin multiplicity due to non-conservation of spin upon preparing the lowest energy singlet products. In such systems, the reaction path leading to the ultimate spin-preserving triplet product is usually barrierless and endoergic with respect to the reactants and is thus thermodynamically unreactive. The first electronically excited state of $\mathrm{O}_{2}$, in contrast, has ${ }^{1} \Delta_{\mathrm{g}}$ symmetry (i.e. singlet spin multiplicity, henceforth ${ }^{1} \mathrm{O}_{2}$ ) and is highly reactive towards most molecular systems due to its thermodynamically favourable and kinetically facile oxidising ability. ${ }^{1}$ Preparation of ${ }^{1} \mathrm{O}_{2}$ is nontrivial, however, since direct electronic excitation from the ground state via, for example, light irradiation is spin-forbidden and therefore improbable. A more viable route for forming ${ }^{1} \mathrm{O}_{2}$ is via indirect photosensitisation using a long-lived chromophore with a photostable triplet state (i.e. via the so-called type II photooxidation). ${ }^{2,3}$ This is the underlying first step in photodynamic

\footnotetext{
${ }^{a}$ Technische Universität München, Lichtenbergstrasse 4,

Garching bei München 85748, Germany. E-mail: tolga.karsili@tum.de

${ }^{b}$ University of Bristol, Cantock's Close, Bristol, BS8 1TS, UK

$\dagger$ Electronic supplementary information (ESI) available. See DOI: 10.1039/c6cc05392k
}

therapy (PDT) $)^{4}$ - which has attracted considerable experimental and theoretical attention. ${ }^{5-8}$

PDT is a form of medicinal treatment in which light induces the photosensitised formation of cellular ${ }^{1} \mathrm{O}_{2}$. The nascent ${ }^{1} \mathrm{O}_{2}$ has been shown to induce cell death - efficiently killing microbial cells. ${ }^{9}$ PDT has also proved effective for the treatment of some forms of cancer - e.g. squamous cell carcinoma (skin cancer). ${ }^{10}$ The mechanisms of PDT have been attributed to ${ }^{1} \mathrm{O}_{2}$-induced oxidation of the molecular structure of cellular DNA that makes up the genetic code - leading to genomic mutations. ${ }^{11,12}$ Though the underlying oxidative mechanism has been theoretically proposed and experimentally established for prototypical systems (e.g. small ethene derivatives ${ }^{13}$ and benzene ${ }^{14}$ ), little is mechanistically known about the activity of ${ }^{1} \mathrm{O}_{2}$ on DNA nucleobases and aromatic amino acids. The feasibility of the His $+\mathrm{O}_{2}$ reaction has previously been detailed using single reference computational methods. ${ }^{17}$ Though the study is extremely informative, ref. 17 lacks important information on the details of the multi-reference character of the potential energy profile associated with the thermally allowed cycloaddition reaction. Our present work also provides information on the role of dispersion upon $\mathrm{His}+\mathrm{O}_{2}$ complexation. In DNA, experiments have shown that the ensuing oxidation reaction is exclusively limited to guanine nucleobases - forming three main intermediates, a mixture of endoperoxide and exoperoxide adducts. ${ }^{15}$ Through thermal molecular rearrangements, these short-lived intermediates form more stable oxoguanine derivatives. Recent theoretical efforts have attempted to understand the underlying reactivity of ${ }^{1} \mathrm{O}_{2}$-induced DNA oxidation ${ }^{16}$ but we are not aware of any prior mechanistic explorations of the topography of the potential energy (PE) profiles involved in the ensuing reaction.

Using highly correlated, multi-reference electronic structure calculations, based on the complete active space with second order perturbation theory (CASPT2) ${ }^{18-20}$ we outline the topography of the PE profiles of ${ }^{1} \mathrm{O}_{2}$-induced oxidation of two prototypical biomolecules: Gua and His. Details of the present calculations are provided in the ESI. $\dagger$ Fig. 1 displays the ground state minimum energy geometries of the prototypical $\pi$-stacked 

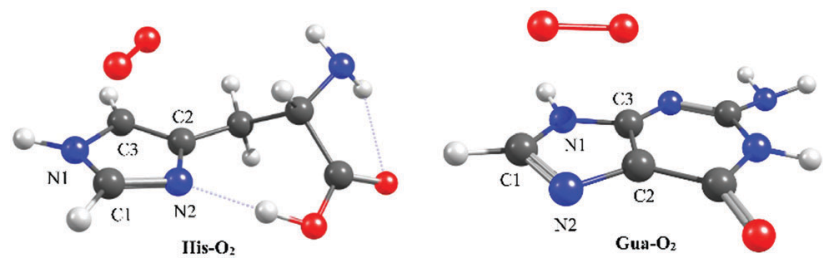

Fig. 1 Minimum energy geometries of the $\pi$-stacked configurations of $\mathrm{His}-\mathrm{O}_{2}$ (left) and $\mathrm{Gua}-\mathrm{O}_{2}$ (right) in their respective ground states. Molecular depictions and Cartesian coordinates of all optimised structures are presented in Fig. S3 of the ESI. $\dagger$

Gua- $\mathrm{O}_{2}$ and His- $\mathrm{O}_{2}$ complexes. These minima were used as the starting points for studying the ensuing [4+2] cycloaddition reaction leading to the endoperoxy intermediate - the thermal reactivity of which is dictated by the Woodward-Hoffmann rules. $^{21}$ Equivalent minima were also located by aligning $\mathrm{O}_{2}$ such that various hydrogen-bonded complexes were formed. Two such low energy hydrogen-bonded complexes were optimised (see Fig. S1 of the ESI $\dagger$ ) but were shown to be less stable than the $\pi$-stacked structure - reflecting the dominance of dispersion effects in $\pi$-stacking. That said, the cellular environment is likely to comprise a mixture of $\pi$-stacked and H-bonded complexes, each of which is likely to lead to reaction of the biomolecule with proximal ${ }^{1} \mathrm{O}_{2}$ dissolved in the cellular solution. Preliminary studies of the lowest energy $\mathrm{H}$-bonded Gua- $\mathrm{O}_{2}$ complexes suggest the facile formation of an exoperoxy intermediate via a coupled addition and hydrogen abstraction reaction. Though important, the formation of the exoperoxy-adduct is beyond the scope of the present work but will form the basis of a fuller future study.

Here we focus on the former reaction in which an endoperoxyintermediate is formed. Fig. 2(a) and (b) present the PE profiles of the three lowest singlet states and the lowest triplet state of the His- $\mathrm{O}_{2} /$ Gua- $\mathrm{O}_{2}$ complex along the $[4+2]$ cycloaddition reaction coordinates (henceforth $Q_{\mathrm{c}[4+2]}$ ) leading to the respective endoperoxyhistidine and endoperoxyguanine cycloadducts. The orbital promotions involved in preparing these states are displayed in Fig. 3(a). In both Gua and His, the $\mathrm{O}_{2}$ moiety adds across the $\mathrm{C} 1$ and $\mathrm{C} 3$ atoms (see Fig. 1 for atomic numberings). The left hand side of Fig. 2(a) and (b) shows the PE profiles linking each state from the respective $\pi$-stacked ground state minimum energy geometry towards the optimised lowest energy transition state (TS) along $Q_{\mathrm{c}[4+2]}$. The right hand panels show results for further calculations, on the lowest singlet potential only, from the TS to the minimum energy configurations of the respective cycloadduct product. The ${ }^{3} \Sigma_{\mathrm{g}}{ }^{-}$and ${ }^{1} \Sigma_{\mathrm{g}}{ }^{+}$electronic states of $\mathrm{O}_{2}$ both contain odd electrons in orthogonal $\pi^{*}$ anti-bonding orbitals, but are distinguishable by the parallel (anti-parallel) arrangement of the respective electron spins (see the molecular orbital diagram in Fig. 3(a)). In isolated $\mathrm{O}_{2}$, the ${ }^{3} \Sigma_{\mathrm{g}}{ }^{-}$and ${ }^{1} \Sigma_{\mathrm{g}}{ }^{+}$electronic states are separated by $\sim 1.6 \mathrm{eV}$, which the present calculations show to increase upon complexation - highlighting the strong dispersion interaction between $\mathrm{O}_{2}$ and the $\pi$-system of Gua/His. Upon cycloaddition with His/Gua, the PEs of the ${ }^{3} \Sigma_{\mathrm{g}}{ }^{-}$and ${ }^{1} \Sigma_{\mathrm{g}}{ }^{+}$ states of $\mathrm{O}_{2}$ (i.e. the pink and red curves) both increase as a
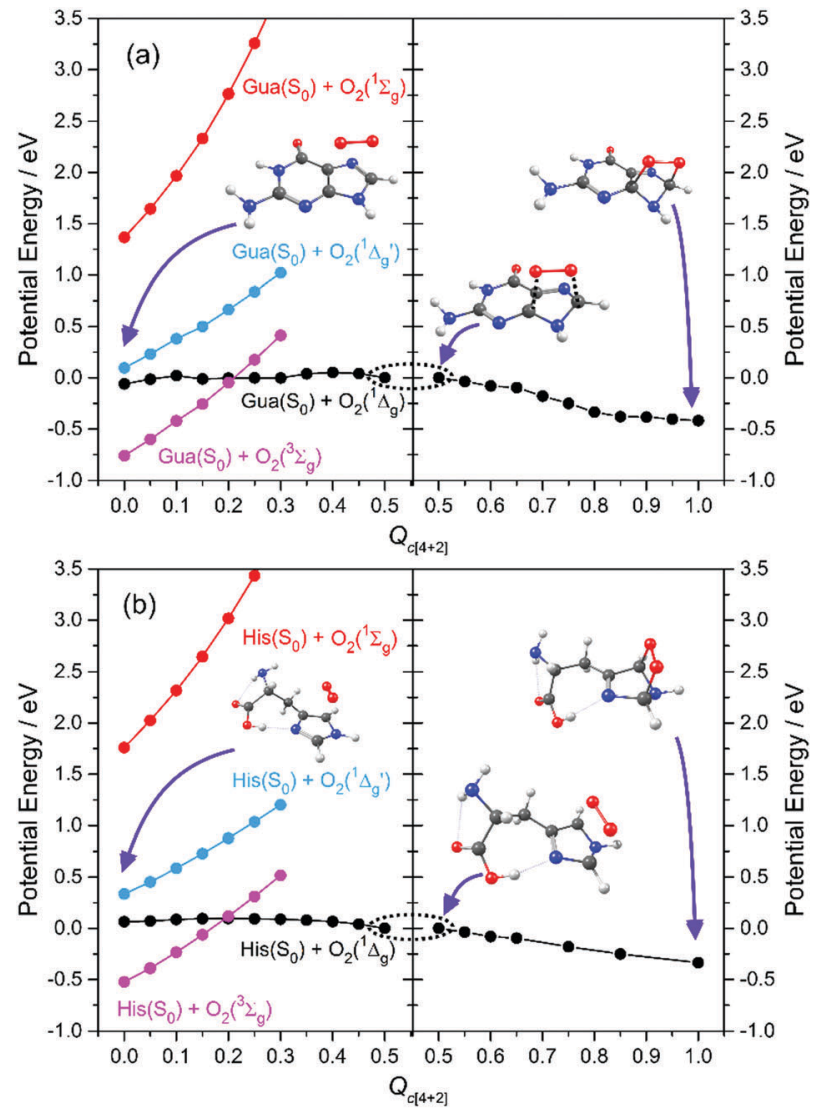

Fig. 2 CASPT2 potential energy profiles along the [4+2] cycloaddition coordinate en route to forming the endoperoxy cycloadduct.

function of $Q_{\mathrm{c}[4+2]}$ - implying an absence of any driving force towards $[4+2]$ cycloaddition. This can be understood by picturing the Woodward-Hoffmann orbital interactions involved in forming cycloadducts with two $\mathrm{C}-\mathrm{O}$ bonds between His/Gua and $\mathrm{O}_{2}$. Since the occupied orbitals of $\mathrm{O}_{2}$ are the highest energy orbitals in Fig. 3(a), formation of the first $\mathrm{C}-\mathrm{O}$ bond requires the cleavage of one of the two bonds in the $\mathrm{O}=\mathrm{O}$ double bond in order to satisfy valency (henceforth step 1). This is achieved by an electron-pair promotion from an occupied $\pi$-orbital of His/Gua to an unoccupied $\pi^{*}$ orbital of $\mathrm{O}_{2}$ - which within the WoodwardHoffmann limit are the optimally aligned $\pi_{\mathrm{His} / \mathrm{Gua}}(1)$ and $\pi^{*} \mathrm{O}_{2}(2)$ orbitals, respectively (see Fig. 3(a)). In much the same way, formation of the second $\mathrm{C}-\mathrm{O}$ bond required to complete the cycloaddition involves promotion of an electron-pair from an occupied $\pi$ orbital of $\mathrm{O}_{2}\left(\pi_{\mathrm{O}_{2}}(1)\right)$ to an unoccupied $\pi^{*}$ orbital $\left(\pi_{\text {His/Gua }}^{*}(1)\right)$ of His/Gua (henceforth step 2).

The relative ordering of the step 1 and 2 electron-pair transfer processes described here is for illustration purposes only. In practice, steps 1 and 2 could occur in any order and in a concerted or stepwise manner - depending on the reaction conditions. Steps 1 and 2 together comprise the overall [4+2] cycloaddition mechanism summarised in Fig. 3(b). Both $\pi^{*}$ orbitals of $\mathrm{O}_{2}$ are already singly occupied in the ${ }^{3} \Sigma_{\mathrm{g}}{ }^{-}$and ${ }^{1} \Sigma_{\mathrm{g}}{ }^{+}$ states, precluding cycloadduct formation between His/Gua and $\mathrm{O}_{2}$ (see Fig. 3(a)). The ${ }^{1} \Delta_{\mathrm{g}}$ state, in contrast, has the two 
(a)

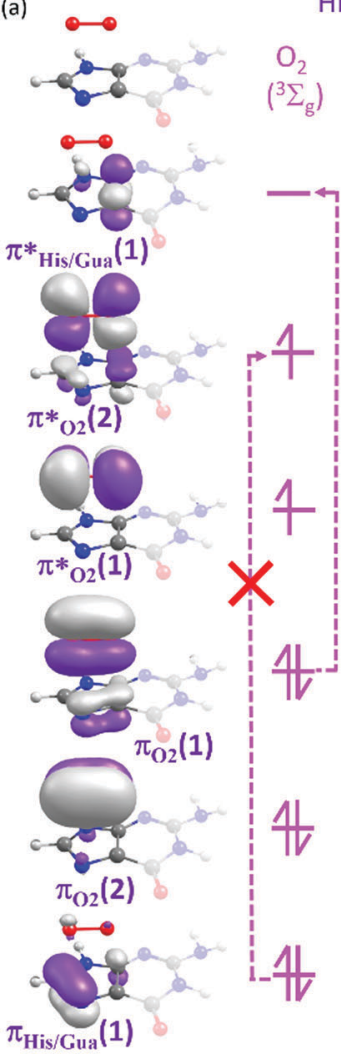

Histidine/Guanine $+\ldots .$.

$\begin{array}{ccc}\mathrm{O}_{2} & \mathrm{O}_{2} & \mathrm{O}_{2} \\ \left({ }^{1} \Sigma_{\mathrm{g}}\right) & \left({ }^{1} \Delta_{\mathrm{g}}\right) & \left({ }^{1} \Delta_{\mathrm{g}}\right)\end{array}$

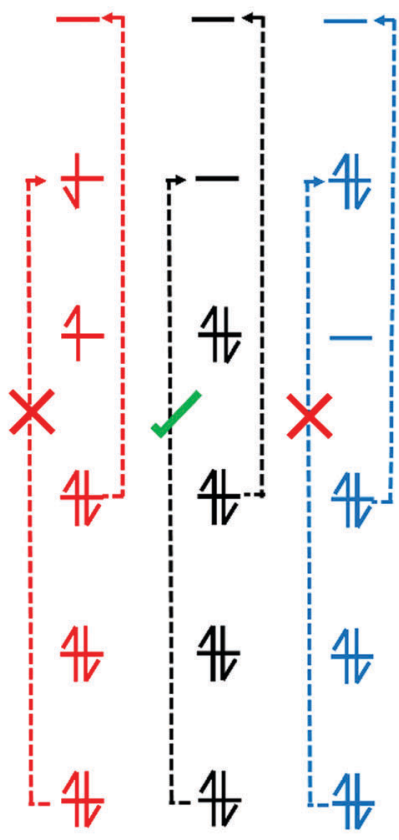

(b) Overall reaction mechanism of Histidine/Guanine $+\mathrm{O}_{2}\left({ }^{1} \Delta_{\mathrm{g}}\right)$ :

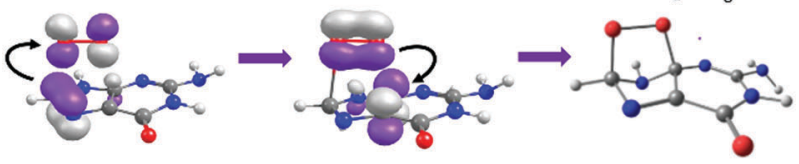

Fig. 3 (a) Selected orbitals and orbital promotions associated with the [4+2] cycloaddition reaction. The dashed vertical arrows with (without) the ticks or crosses represent steps 1 (2) of the cycloaddition reaction, with the tick/cross indicating, respectively, an allowed/forbidden electron-pair transfer in step 1. (b) A simplified mechanism summarizing the electronpair migrations (curly black arrows) associated with the [4+2] cycloaddition reaction with ${ }^{1} \mathrm{O}_{2}$.

electrons spin-paired in one of the two $\pi^{*}$ orbitals. These $\pi^{*}$ orbitals are degenerate in an isolated $\mathrm{O}_{2}$ molecule, and each has the same probability of containing the electron-pair. In isolated $\mathrm{O}_{2}$, this doubly degenerate ${ }^{1} \Delta_{\mathrm{g}}$ state is $\sim 1 \mathrm{eV}$ above that of the ${ }^{3} \Sigma_{\mathrm{g}}{ }^{-}$state. The present calculations show a decrease in the ${ }^{3} \Sigma_{\mathrm{g}}{ }^{-}{ }^{1} \Delta_{\mathrm{g}}$ energy gap upon $\mathrm{O}_{2}+$ His/Gua complexation. Within this complex the degeneracy in the ${ }^{1} \Delta_{\mathrm{g}}$ state must be lifted, since the two $\pi^{*}$ orbitals will interact differently when in close proximity to His/Gua. This lifting of the degeneracy is evident in Fig. 2(a) and (b) (as the $\mathrm{O}_{2}$ moiety approaches His/ Gua) - manifesting in two PE curves describing the Gua $\left(\mathrm{S}_{0}\right) /$ $\operatorname{His}\left(\mathrm{S}_{0}\right)+\mathrm{O}_{2}\left({ }^{1} \Delta_{\mathrm{g}}\right)$ configuration (i.e. black and blue curves in Fig. 2 - henceforth ${ }^{1} \Delta_{\mathrm{g}}$ and $\left.{ }^{1} \Delta_{\mathrm{g}}{ }^{\prime}\right)$ - the lower energy of which (black curve) is reactive with respect to $Q_{\mathrm{c}[4+2]}$.

We also note that the Gua $\left(\mathrm{S}_{0}\right) / \mathrm{His}\left(\mathrm{S}_{0}\right)+\mathrm{O}_{2}\left({ }^{1} \Delta_{\mathrm{g}}\right)$ and $\mathrm{Gua}\left(\mathrm{S}_{0}\right) /$ $\mathrm{His}\left(\mathrm{S}_{0}\right)+\mathrm{O}_{2}\left({ }^{3} \Sigma_{\mathrm{g}}{ }^{-}\right)$potentials cross en route to the lowest energy TS - thereby providing a route by which ${ }^{1} \mathrm{O}_{2}$ could relax to reform ${ }^{3} \mathrm{O}_{2}$. This relaxation will be determined by the rate of intersystem crossing (ISC) which is likely to be slower (nanosecond or longer timescale) than the typical thermal (picosecond timescale) motions of the nuclei that are expected to drive the cycloaddition reaction. Thus, though we recognise that some population evolving on the Gua $\left(\mathrm{S}_{0}\right) / \mathrm{His}\left(\mathrm{S}_{0}\right)+\mathrm{O}_{2}\left({ }^{1} \Delta_{\mathrm{g}}\right)$ potential could branch at this crossing, we anticipate that spinorbit coupling will be relatively weak and that cycloadduct formation would be the dominant process. Notwithstanding, under favourable reaction conditions, the ${ }^{3} \mathrm{O}_{2}+\mathrm{His} / \mathrm{Gua}$ limit could undergo a spin-flipping reaction by ISC to the ${ }^{1} \mathrm{O}_{2}+$ His/ Gua state at this crossing.

Beyond the TS, the lower energy Gua $\left(\mathrm{S}_{0}\right) / \mathrm{His}\left(\mathrm{S}_{0}\right)+\mathrm{O}_{2}\left({ }^{1} \Delta_{\mathrm{g}}\right)$ potential continues to decline in energy as it evolves to the endoperoxy cycloadduct. The $\mathrm{Gua}\left(\mathrm{S}_{0}\right) / \mathrm{His}\left(\mathrm{S}_{0}\right)+\mathrm{O}_{2}\left({ }^{1} \Delta_{\mathrm{g}}{ }^{\prime}\right)$ potential (blue curve), in contrast, increases in energy along $Q_{\mathrm{c}[4+2]}$. This stark difference in the reactivity of the ${ }^{1} \Delta_{\mathrm{g}}$ and ${ }^{1} \Delta_{\mathrm{g}}{ }^{\prime}$ states of $\mathrm{O}_{2}$ with Gua/His can also be understood by considering the orbitals involved in preparing the cycloadduct. The favourable driving force for cycloaddition in the lower energy case can be understood by recognising that the optimally aligned $\pi_{\text {His/Gua }}(1)$ donor and $\pi^{*} \mathrm{O}_{2}(2)$ acceptor orbitals are, respectively, occupied and unoccupied - as required for the step 1 electron-pair transfer. In the case of the higher energy $\operatorname{Gua}\left(\mathrm{S}_{0}\right) / \mathrm{His}\left(\mathrm{S}_{0}\right)+\mathrm{O}_{2}\left({ }^{1} \Delta_{\mathrm{g}}{ }^{\prime}\right)$ encounter, however, the optimally aligned Woodward-Hoffmann $\pi_{\mathrm{His} / \mathrm{Gua}}(1)$ and $\pi^{*} \mathrm{O}_{2}(2)$ orbitals are both doubly occupied (see Fig. 3(a)). Hence, the step 1 electron-pair transfer is unfavourable, as shown by the increase in PE along $Q_{\mathrm{c}[4+2]}$.

At this point, we note that the present calculations are limited to the isolated 'gas phase' complexes and that we do not attempt to include any of the additional complexities associated with the surrounding cellular environment that would be necessary for a more complete biochemical study. The present topographies of the returned PE profiles may also depend on the electrostatic embedding in the bulk DNA environment. That said, the present calculations are nonetheless very informative, as they illustrate the PE profiles associated with the experimentally observed [4+2] cycloaddition of ${ }^{1} \mathrm{O}_{2}$ to the chosen biomolecular constituent. More specifically, we have used highly correlated multi-reference electronic structure methods to determine PE profiles for the most widely recognised form of biomolecular oxidation via ${ }^{1} \mathrm{O}_{2}$ : [4+2] cycloaddition of $\mathrm{O}_{2}$ to His/Gua. In so doing, we have shown that the ${ }^{1} \Delta_{\mathrm{g}}$ state of isolated $\mathrm{O}_{2}$ splits into two non-degenerate electronic states when $\pi$-stacked with a conjugated biomolecule the lower of which is the sole state that is reactive towards cycloaddition in the low energy $\pi$-stacked configuration shown in Fig. 1. The hitherto neglected higher energy ${ }^{1} \Delta_{\mathrm{g}}{ }^{\prime}$ state of $\mathrm{O}_{2}$ is unreactive with ground state His/Gua along $Q_{\mathrm{c}[4+2]}$, but this state also possesses a vacant $\pi^{*}$ orbital that is orthogonal to the coordinate along which His/Gua and $\mathrm{O}_{2}$ moieties $\pi$-stack. The higher energy ${ }^{1} \Delta_{\mathrm{g}}$ ' state is thus likely to be reactive with proximal aromatic molecules that happen to stack in the correct orientation highlighting the importance of structure and orientation in the reactivity of ${ }^{1} \mathrm{O}_{2}$ with an associated (bio)molecule.

The cycloadducts shown to the far right of Fig. 2(a) and (b) constitute an intermediate that is likely to undergo further 
rearrangement to form various more stable products. Recent theoretical studies by Dumont et al. ${ }^{16}$ have identified possible products on the ground state potential including 4-hydroxy-8oxo-deoxyguanine and 8-oxoguanine and spironucleoside derivatives. Most of these products were shown to be very exoergic with respect to the endoperoxy cycloadduct. Within cellular DNA, the eventual formation of such products is likely to lead to irreversible DNA damage - ultimately rendering the normal working functions of the cell inactivated. This is particularly desirable for toxic (e.g. cancerous) cells. As noted, the above experiments have shown that the reactivity of ${ }^{1} \mathrm{O}_{2}$ is exclusively limited to Gua. Comparing the available experiments on the purine and pyrimidine bases, it is clear that the presently studied [4+2] cycloaddition reaction favours the five-membered imidazole constituent of the bicyclic purine systems. This can be simply understood by considering that the five-membered imidazole ring constituent provides an optimal C1-C3 bond distance and $\pi$-electron distribution for a thermally allowed [4+2] cycloaddition reaction whilst the sixmembered ring constituent does not. With the unavailability of a five-membered ring, the unreactivity of pyrimidine bases is therefore plausibly attributable to the larger $\mathrm{C}-\mathrm{C}$ bonding distances and, in the case of uracil and thymine, the unfavourable distribution of $\pi$-electrons in order to sustain a $[4+2]$ cycloaddition. Notwithstanding, the possibility of a $[4+2]$ cycloaddition between cytosine and ${ }^{1} \mathrm{O}_{2}$ exists - though large reaction barriers hinder this process (see Fig. S2 of the ESI $\dagger$ ). Comparing the reactivity of guanine with that of adenine is less trivial since (to first order) both contain an imidazole constituent with an optimal distribution of $\pi$-electrons. Dumont et al. went some way to explaining this trend by showing that the reaction barrier associated with the ${ }^{1} \mathrm{O}_{2}+$ adenine cycloaddition reaction was larger than that for guanine - though this too highlights the importance of a mechanistic study of the PE profile.

Therefore, understanding the topography of the PE surface of such oxidative reactions could be insightful not least for studying the effect of other reactive species (oxygen or otherwise) potentially aiding in the manufacturing of photosensitisers that enhance the formation yield of such species. The present work adds to the small but growing number of reported mechanistic studies on the oxidation of biomolecules by ${ }^{1} \mathrm{O}_{2}$. Given the continued advances in both theoretical and experimental methods, many more such studies can be expected in the near future - involving ${ }^{1} \mathrm{O}_{2}$ or other potentially reactive intermediates with (bio)molecules.

The authors thank Prof. M. N. R. Ashfold (University of Bristol) for fruitful discussions and the EPSRC (EP/L005913) and TUM for funding. The data underpinning the present study has been stored in the University of Bristol research data repository and can be accessed via the following link: DOI: 10.5523/bris.qsf82vq3qbm31kac5ggbn4ldx.

\section{Notes and references}

1 R. Y. N. Ho, J. F. Liebman and J. S. Valentine, in Active Oxygen in Chemistry, ed. C. S. Foote, J. S. Valentine, A. Greenberg and J. F. Liebman, Springer Netherlands, Dordrecht, 1995, pp. 1-23.

2 C. Schweitzer and R. Schmidt, Chem. Rev., 2003, 103, 1685-1758.

3 A. Greer, Acc. Chem. Res., 2006, 39, 797-804.

4 J. Chen, L. Keltner, J. Christophersen, F. Zheng, M. Krouse, A. Singhal and S-s. Wang, Cancer J., 2002, 8, 154-163.

5 E. Skovsen, J. W. Snyder, J. D. C. Lambert and P. R. Ogilby, J. Phys. Chem. B, 2005, 109, 8570-8573.

6 M. Klaper, W. Fudickar and T. Linker, J. Am. Chem. Soc., 2016, 138, 7024-7029.

7 M. Klaper and T. Linker, J. Am. Chem. Soc., 2015, 137, 13744-13747.

8 S. Kim, T. Tachikawa, M. Fujitsuka and T. Majima, J. Am. Chem. Soc., 2014, 136, 11707-11715.

9 M. R. Hamblin and T. Hasan, Photochem. Photobiol. Sci., 2004, 3, 436-450.

10 J. Chen, L. Keltner, J. Christophersen, F. Zheng, M. Krouse, A. Singhal and S.-s. Wang, Cancer J., 2002, 8, 154-163.

11 T. P. A. Devasagayam, S. Steenken, M. S. W. Obendorf, W. A. Schulz and H. Sies, Biochemistry, 1991, 30, 6283-6289.

12 J. Piette, J. Photochem. Photobiol., B, 1991, 11, 241-260.

13 T. Saito, S. Nishihara, Y. Kataoka, Y. Nakanishi, Y. Kitagawa, T. Kawakami, S. Yamanaka, M. Okumura and K. Yamaguchi, J. Phys. Chem. A, 2010, 114, 7967-7974.

14 A. G. Leach and K. N. Houk, Chem. Commun., 2002, 1243-1255.

15 J.-L. Ravanat and J. Cadet, Chem. Res. Toxicol., 1995, 8, 379-388.

16 E. Dumont, R. Grüber, E. Bignon, C. Morell, Y. Moreau, A. Monari and J.-L. Ravanat, Nucleic Acids Res., 2015, 44, 56-62.

17 J. Méndez-Hurtado, R. López, D. Suárez and M. I. Menéndez, Chem. - Eur. J., 2012, 18, 8437-8447.

18 B. O. Roos and K. Andersson, Chem. Phys. Lett., 1995, 245, 215-223. 19 T. H. Dunning, Jr., J. Chem. Phys., 1989, 90, 1007-1023.

20 H. J. Werner, P. J. Knowles, G. Knizia, F. R. Manby, M. Schütz, P. Celani, T. Korona, R. Lindh, A. Mitrushenkov, G. Rauhut, K. R. Shamasundar, T. B. Adler and R. D. Amos, et al., MOLPRO, University of Cardiff, Cardiff, U.K., 2010.

21 R. B. Woodward and R. Hoffmann, J. Am. Chem. Soc., 1965, 87, 395-397. 